\title{
Clutter filtering issues in speckle tracking for two-dimensional blood velocity estimation
}

\section{- a potential solution based on compounded imaging}

\author{
Solveig Søvik Alnes*, Abigail Swillens ${ }^{\ddagger}$, Patrick Segers ${ }^{\ddagger}$, Hans Torp* and Lasse Lovstakken*† \\ ${ }^{*}$ MI Lab and Dept. of Circulation and Medical Imaging, NTNU, Trondheim, Norway \\ ${ }^{\dagger}$ St. Olavs Hospital, Trondheim, Norway \\ ${ }^{\ddagger}$ IBITech-BioMMeda Ghent University, Belgium
}

\begin{abstract}
Clutter filtering issues remains a major limitation in multi-dimensional blood velocity estimation. In this work we investigate how compounded plane wave imaging can be used to minimize clutter filtering issues when using speckle tracking for two-dimensional velocity estimation. By using a dual scan angle approach one can ensure a sufficient beam-to-flow angle for most imaging scenarios. Segmentation algorithms based on the estimated power and mean axial velocity were used to determine whether estimates were to be discarded or combined. The proposed setup was evaluated in a simulation study (Field II) for straight tube flow with parabolic flow profiles, and for a realistic CFD flow model of a carotid bifurcation. It was shown that the clutter filtering issues were significantly reduced when using compounded imaging and speckle tracking. Regions corrupted by the clutter filter in one scan was not corrupted in the other scan. Thus, consistent estimates could be obtained from the whole region of interest. In addition, the estimates were averaged and the variance reduced in regions where neither scan was corrupted. Regression analysis showed a 10-16 \% decrease in standard deviation for the lateral velocity component and a $17-19 \%$ decrease for the axial component.
\end{abstract}

\section{INTRODUCTION}

Ultrasound imaging is a frequently used non-invasive tool for blood flow visualization. The conventional Doppler methods have been extensively validated and are used in a wide range of clinical settings today. However, since only the velocity component along the beam direction is measured, blood flow perpendicular to the beam will not be imaged. General improvements of the imaging methods would minimize the need of interpreting the color images, and therefore increase the diagnostic certainty. Several flow velocity estimators for estimating both the axial and lateral velocity component have been proposed. Speckle tracking[1] and vector-Doppler[2] are the two main research lines for two-dimensional velocity estimation, but lately new methods as echo PIV and digital processing of color-Doppler information are also suggested [3],[4]. High-pass filtering is still necessary to remove the strong signal from the surrounding tissue and this remains a major limitation in multi-dimensional blood velocity estimation. Since clutter filtering is currently based on slow-time wall filtering as in conventional color flow imaging(CFI), blood velocity estimates are still corrupted for near-perpendicular beam-to-flow angles. The clutter filter frequency response determines the lower limit regarding detection and estimation of blood velocities. For approaches based on the autocorrelation method, a bias is induced in the filter transition region. For speckle tracking, both the power and the bandwidth of the blood signal is decreased when parts of the signal is under the filter cut-off. As a result of this, the speckle will be smeared out and speckle correlation is degraded[5]. In addition, speckle decorrelates rapidly due to spatial and temporal velocity gradients, so high frame rates are essential. Recent developments to increase acquisition rates are based on transmitting plane waves and utilizing parallel receive beamforming(PRB)[6]. Such scan setups make it possible to achieve high frame rates even when increasing slow time ensemble lengths.

In this work we investigate how compounded plane wave imaging(PWI) can be used to minimize clutter filtering issues when using speckle tracking for two-dimensional blood velocity estimation. In speckle tracking, only one scan angle is needed to produce an image with two-dimensional velocity estimates. A second tilted scan may therefore be added to avoid a perpendicular beam-to-flow angle for all areas in the region of interest. Firstly, we investigated clutter filter influence on the velocity estimates from speckle tracking. Results from these simulations were used to design a scan sequence of two opposite tilted scans at minimum angles in order to avoid critical beam-to-flow angles. Minimum tilt angles are desired as they minimize loss of imaging depth. Secondly, the developed compounded plane wave setup was evaluated in simulations of a carotid bifurcation based on computational fluid dynamics(CFD).

\section{Methods}

\section{A. Data acquisition}

The proposed setup is a two-angle interleaving plane wave scan sequence, as sketched in figure 1. Plane waves on transmit and parallel beamforming on receive is utilized to achieve high frame rates and instantaneous images. With this setup, a high ensemble size, meaning more samples available for processing, and a high frame rate is achievable.

\section{B. Clutter filtering}

The strong signal from the surrounding tissue must be filtered out before one can do speckle tracking on the blood signal. Potential clutter filters are finite impulse response (FIR) 


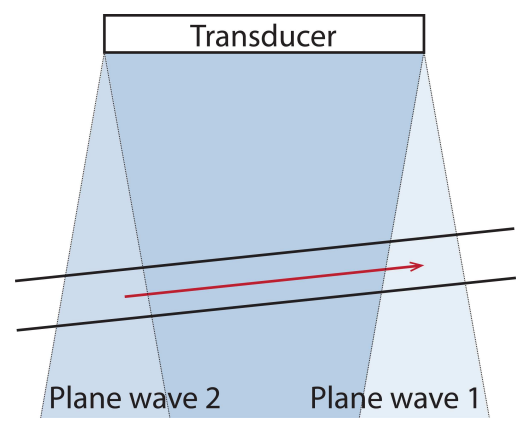

Fig. 1. Scan setup with two transmit angles.

TABLE I

SIMULATION PARAMETERS

\begin{tabular}{|c||c|}
\hline Probe type & 192 element linear array \\
Pulse center frequency & $5 \mathrm{MHz}$ \\
Transmit aperture & $4.72 \mathrm{~cm}$ (full aperture) \\
Receive F-number & 1.4 \\
PRF & $4 \mathrm{kHz}$ \\
Ensemble size & $20-60$ \\
SNR & 30 \\
\hline
\end{tabular}

filters, infinite impulse response (IIR) filters and polynomial regression filters. Since speckle tracking depends on the correlation of the speckle in subsequent ensemble samples, a time-invariant filter, i.e a FIR filter, is preferred. In this work a fourth order minimum phase FIR filter with cut-off at $0.33 * V_{\text {Nyquist }}$ has been used. Due to filter initialization, the first four samples were discarded in the filter output.

\section{Speckle tracking}

In speckle tracking a kernel region is identified in an image acquisition and then the best match of this kernel is searched for in a subsequent acquisition. In this work the best match was found using the sum-of-absolute-differences (SAD) algorithm and this determines the velocity vector of the kernel. When this is done for all points in the image and the estimates are averaged over the ensemble size, a vector map of the estimated velocities is obtained. Spatial averaging is required to reduce the variance of the estimates to an acceptable level.

The influence of the clutter filter on speckle tracking performance was first studied in straight tube simulations using Field II[7]. The simulated scatterers followed parabolic velocity profiles with varying Doppler angles. The tube had a radius $2.5 \mathrm{~cm}$ and was tilted to gradually move the signal from blood into the filter transition and stop band region. The parabolic flow profile had a maximum velocity of $0.5 \mathrm{~m} / \mathrm{s}$.

The compounded plane wave setup was simulated using a realistic carotid bifurcation model. Here the scatterers follow the realistic flow field simulated using CFD[8]. This gives us the opportunity to compare the estimated velocities with the reference CFD flow field. The simulations were carried out for ensemble lengths of 20 and tilted scan angles from \pm 5 to \pm 10 degrees. The simulation parameters are listed in table I.
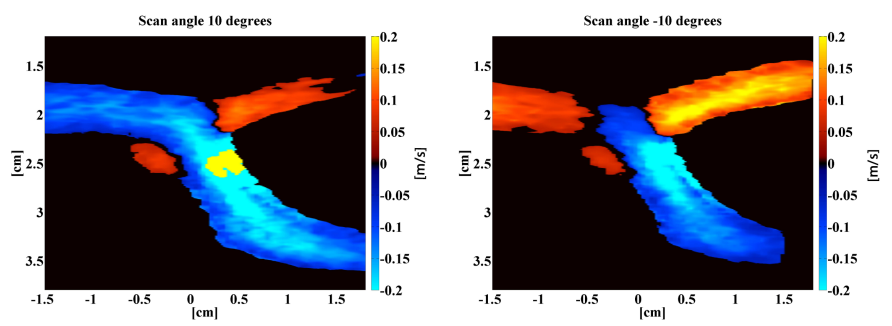

Fig. 2. Segmentation maps for compounding based on color flow imaging.

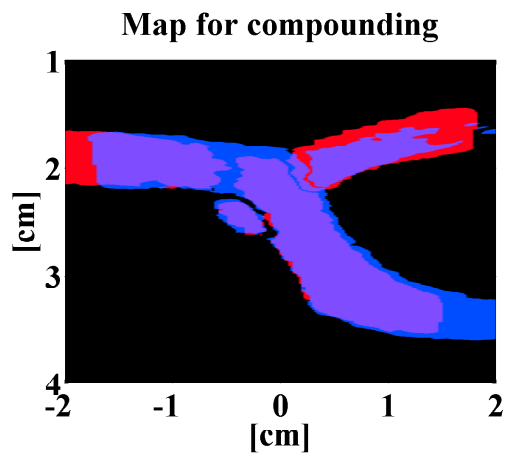

Fig. 3. Purple represents the region where the estimates have been combined. Blue region represents estimates from scan angle $10^{\circ}$ and the red region represents estimates from scan angle $-10^{\circ}$.

\section{Segmentation algorithm}

A velocity vector map is obtain for each scan angle, so a combined velocity vector map can be put together by either combining or discarding the estimates. Using a segmentation algorithm based on the estimated power and mean axial velocity, we obtain an arbitration map for each scan angle where regions corrupted by the clutter filter are segmented away. Based on these arbitration maps the velocity estimates are either averaged with the estimate from the other scan angle or discarded.

The segmentation algorithm used for the color flow images in figure 2 forms the arbitration map shown in figure 3 for combining or discarding estimates. The region where the estimates are combined is indicated with purple. In the blue region the estimates from scan angle $-10^{\circ}$ are discarded, whereas the estimates from scan angle $10^{\circ}$ is discarded in the red region.

\section{RESUlTS}

\section{A. Straight tube simulations}

In figure 4 , the bias of the estimated axial velocity from speckle tracking is compared to the autocorrelation method. It is observed that speckle tracking gives reasonable estimates in the clutter filter transition region where the autocorrelation method gives significant bias. In the filter transition region the autocorrelation method starts to overestimate due to the attenuation of lower frequencies. For speckle tracking, the velocity estimate is underestimated with a higher variance than for the autocorrelation method, but with a significantly lower bias in the filter transition region. 


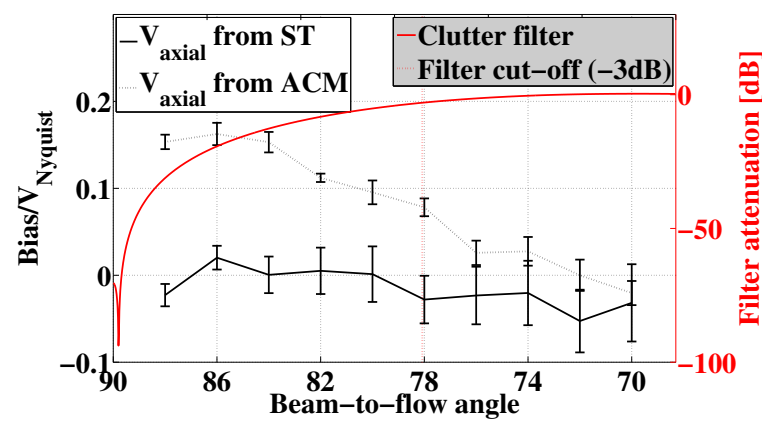

Fig. 4. Bias of estimated axial velocity from speckle tracking and the autocorrelation method for beam-to-flow angle of 70-88 degrees. Signal-tonoise ratio is $30 \mathrm{~dB}$.

\section{B. Carotid bifurcation simulations}

In the scatterplots in figure 5 the estimated axial $\left(V_{z}\right)$ and lateral $\left(V_{x}\right)$ velocity components from a simulation with packet size 20 and scan angles $\pm 10^{\circ}$ are compared with the reference velocity field from CFD. A higher variance is observed for the lateral component than the axial for both scan angles. In addition we observe a higher variance among the velocities which are below the filter cut off for the lateral component. In the bottom two scatterplots the compounded velocity components are compared with the reference. Here we observe a lower variance and a better consistency than in the estimates from each scan. The analysis showed a 10$16 \%$ decrease in standard deviation for the lateral velocity component and a $17-19 \%$ decrease for the axial component.

\section{DISCUSSION}

In this work we show that the clutter filtering issues can be reduced using a dual-scan sequence. From straight tube simulations we could see that speckle tracking provided reasonable estimates well below the filter cut-off. These results indicated that we can have small tilt angles in our proposed scan setup and still avoid critical beam-to-flow angles for speckle tracking. We can therefore minimize the clutter filtering issues without significantly compromising the imaging depth. However, the setup is dependent on transmission of plane waves to achieve high frame rates and increase the accuracy of speckle tracking. Since plane waves have reduced penetration depth compared to focused waves, this will also be a limiting factor of the imaging depth.

In the setup, estimates were discarded in all areas corrupted by the filter, but the corrupted regions were not overlapping for the two scans. Thus, in a dual-scan setup, consistent information could be obtained in the whole region of interest. The estimates were averaged in the regions where neither scan had been corrupted by the clutter filter, so that the variance in these regions was reduced.

\section{CONClusion}

Using two oppositely tilted scans to avoid critical beamto-flow angles, we could significantly reduce clutter filtering
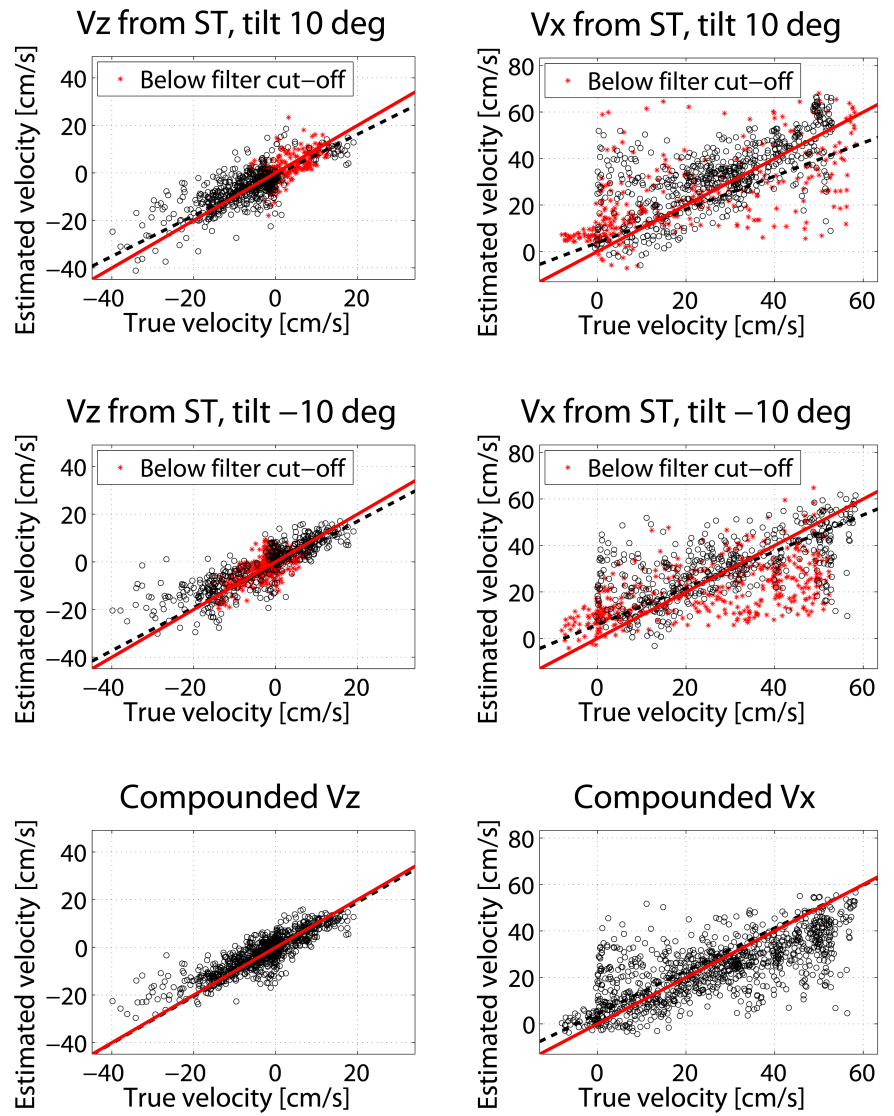

Fig. 5. In the scatterplots the ST estimates for the axial and lateral velocity components from the two scan angles and the compounded velocities are compared to the true velocity field from CFD.

issues when using speckle tracking for two-dimensional blood velocity estimation. As speckle tracking gave consistent estimates well into the clutter filter transition region, this can be achieved without substantially compromising scan depth.

\section{REFERENCES}

[1] L.N. Bohs, B.J. Geiman, M.E. Anderson, S.C. Gebhart and G.E. Trahey, "Speckle tracking for multi-dimensional flow estimation," Ultrasonics, vol. 38, pp. 369-375, 2000.

[2] B. Dunmire, K.W. Beach, K-H. Labs, M. Plett and D.E. Strandness Jr., "Cross-beam vector Doppler ultrasound for angle-independent velocity measurements," Ultrasound Med. Biol., vol. 26, pp. 1213-1235, 2000.

[3] H.B. Kim, J.R. Hertzberg and R. Shandas, "Development and validation of echo PIV," Experiments in Fluids, vol. 36, pp. 455-462, 2004.

[4] D. Garcia, et. al., "Two-dimensional intraventricular flow mapping by digital processing conventional color-Doppler echocardiography images," IEEE Trans Med Imaging, vol. 29, pp. 1701-1713, 2010.

[5] S. Bjærum, "Detection and visualization of moving targets in medical ultrasound imaging, paper H: Blood Motion Imaging: A new blood flow imaging technique," Ph.D. dissertation, Norwegian University of Science and Technology, Trondheim, Norway, 2001.

[6] J. Udesen, et. al., "High Frame-Rate Blood Vector Velocity Imaging Using Plane Waves: Simulations and Preliminary Experiments," IEEE Trans. Ultrason., Ferroelect., Freq. Contr., vol. 55, pp. 1729-1743, 2008.

[7] J.A. Jensen, "Field: A program for simulating ultrasound systems," Med. Biol. eng. Comput., vol. 34, pp. 351-352, 1996.

[8] A. Swillens, L. Lovstakken, J. Kips, H. Torp and P. Segers, "Ultrasound simulation of complex flow velocity fields based on computational fluid dynamics" IEEE Trans. Ultrason., Ferroelect., Freq. Contr., vol. 56, pp. 204-216, 2009 